\title{
EXTENSIONS OF $*$-REPRESENTATIONS
}

\author{
ANDREAS KASPAREK
}

(Communicated by Paul S. Muhly)

\begin{abstract}
Let $\pi$ be a *-representation of a $*$-algebra $\mathfrak{A}$. In general the strong commutant $\pi(\mathfrak{A})_{s}^{\prime}$ and the weak commutant $\pi(\mathfrak{A})_{w}^{\prime}$ of the $\mathscr{O}^{*}$-algebra $\pi(\mathfrak{A})$ do not coincide. We are looking for some methods to get extensions of $\pi$ such that the related commutants coincide or which are even selfadjoint. In $\S \S 2$ and 3 we consider so-called generated extensions that are a modification of induced extensions investigated by Borchers, Yngvason [1] and Schmüdgen [7]. In $\S 4$ let $\mathfrak{A}$ be a $*$-algebra and $\mathfrak{B}$ a subset of its hermitian part $\mathfrak{A}_{h}$ such that $\mathfrak{A}$ is generated by $\mathfrak{B} \cup\{1\}$ as an algebra. We present a method to extend *-representations $\pi$ of such algebras, which is closely related with the extension of the symmetric operators $\pi(b), b \in \mathfrak{B}$. In $\S 5$ we give an example that shows that the method of generated extensions is also suitable to get extensions such that the commutants of the related $\mathscr{O}^{*}$-algebras coincide.
\end{abstract}

\section{Preliminaries}

We begin with some general notational conventions. $\mathbb{N}$ and $\mathbb{N}_{0}$ are the positive and nonnegative integers, respectively. The scalar product of a Hilbert space $\mathscr{H}$ is denoted by $\langle\cdot, \cdot\rangle$. It is supposed to be linear with respect to the first variable and antilinear with respect to the second variable. Let $A$ be a linear operator in a Hilbert space $\mathscr{H}$. We denote by $\mathscr{D}(A)$ the domain, by $\mathscr{R}(A)$ the range, by $\bar{A}$ the closure, and by $A^{*}$ the adjoint of $A$. If $A$ is closed then $\mathscr{D} \subseteq \mathscr{D}(A)$ is called a core for $A$ if the restriction $A \mid \mathscr{D}$ of $A$ to $\mathscr{D}$ satisfies $\overline{A \uparrow \mathscr{D}}=A$.

Let $A$ be a closed symmetric operator in a Hilbert space $\mathscr{H}$. Then,

$$
(A)_{s}^{\prime}:=\{C \in \mathbb{B}(\mathscr{H}): C \mathscr{D}(A) \subseteq \mathscr{D}(A) \text { and } C A \varphi=A C \varphi
$$

$$
\text { for all } \varphi \in \mathscr{D}(A)\}
$$

is called the strong commutant of $A$ and

$$
(A)_{w}^{\prime}:=\left\{C \in \mathbb{B}(\mathscr{H}): C \mathscr{D}(A) \subseteq \mathscr{D}\left(A^{*}\right) \text { and } C A \varphi=A^{*} C \varphi\right.
$$

$$
\text { for all } \varphi \in \mathscr{D}(A)\}
$$

is called the weak commutant of $A$.

Received by the editors July $10,1989$.

1980 Mathematics Subject Classification (1985 Revision). Primary 47D40; Secondary 46K10.

Key words and phrases. Extensions of $*$-representations, commutants of $\mathscr{O}^{*}$-algebras. 
We now recall some definitions about unbounded operator algebras and $*$ representations. Let $\mathscr{D}$ be a dense linear subspace of a Hilbert space $\mathscr{H}$. An $\mathscr{O}^{*}$-algebra $\mathscr{A}$ on $\mathscr{D}$ is a $*$-algebra of linear operators defined on $\mathscr{D}$ and leaving $\mathscr{D}$ invariant, which contains the identity map $I$ of $\mathscr{D}$. The multiplication in $\mathscr{A}$ is the composition of the operators and the involution in $\mathscr{A}$ is the map $A \rightarrow A^{+}:=A^{*} \mid \mathscr{D}$. The graph topology of $\mathscr{A}$ is the locally convex topology $t_{\mathscr{A}}$ on $\mathscr{D}$, which is defined by the seminorms $\|\cdot\|_{A}:=\|A \cdot\|$, $A \in \mathscr{A}$.

Let $\mathfrak{A}$ be an abstract $*$-algebra with unit element 1 . A *-representation of $\mathfrak{A}$ on $\mathscr{D}$ (dense in a Hilbert space $\mathscr{H}$ ) is a $*$-homomorphism $\pi$ of $\mathfrak{A}$ onto an $\mathscr{O}^{*}$. algebra on $\mathscr{D}$, which maps 1 into the identity map on $\mathscr{D}$. Then, $\mathscr{D}(\pi):=\mathscr{D}$ is called the domain of $\pi$, and we write $\mathscr{H}(\pi):=\mathscr{H}$. Let $\pi_{1}$ and $\pi_{2}$ be *representations of a $*$-algebra $\mathfrak{A} . \pi_{2}$ is an extension of $\pi_{1}$ if $\mathscr{H}\left(\pi_{1}\right) \subseteq \mathscr{H}\left(\pi_{2}\right)$, $\mathscr{D}\left(\pi_{1}\right) \subseteq \mathscr{D}\left(\pi_{2}\right)$, and $\pi_{1}(a)=\pi_{2}(a) \uparrow \mathscr{D}\left(\pi_{1}\right)$ for all $a \in \mathfrak{A}$. A *-representation $\pi$ of $\mathfrak{A}$ is closed if the locally convex space $\mathscr{D}\left[t_{\pi(\mathfrak{a})}\right]$ is complete. This is true if and only if $\mathscr{D}=\bigcap_{a \in \mathfrak{A}} \mathscr{D}(\overline{\pi(a)})$. The adjoint of a $*$-representation $\pi$ is given by $\mathscr{D}\left(\pi^{*}\right):=\bigcap_{a \in \mathfrak{A}} \mathscr{D}\left(\pi(a)^{*}\right), \pi^{*}(a):=\pi\left(a^{+}\right)^{*} \mid \mathscr{D}\left(\pi^{*}\right)$ for all $a \in \mathfrak{A}$. A $*$-representation $\pi$ is called selfadjoint if $\pi=\pi^{*}$. The latter is true if and only if $\mathscr{D}\left(\pi^{*}\right)=\mathscr{D}$.

Let $\mathscr{A}$ be an $\mathscr{O}^{*}$-algebra on $\mathscr{D}$. Define

$$
\mathscr{A}_{s}^{\prime}:=\{C \in \mathbb{B}(\mathscr{H}): C \mathscr{D} \subseteq \mathscr{D} \text { and } C A \varphi=A C \varphi
$$

for all $\varphi \in \mathscr{D}$ and $A \in \mathscr{A}\}$

and

$$
\begin{aligned}
& \mathscr{A}_{w}^{\prime}:=\left\{C \in \mathbb{B}(\mathscr{H}):\langle C A \varphi, \psi\rangle=\left\langle C \varphi, A^{+} \psi\right\rangle \text { for all } \varphi, \psi \in \mathscr{D}\right. \\
&\text { and } A \in \mathscr{A}\} .
\end{aligned}
$$

$\mathscr{A}_{s}^{\prime}$ is called the strong commutant and $\mathscr{A}_{w}^{\prime}$ the weak commutant of the $\mathscr{O}^{*}$ algebra $\mathscr{A}$. Obviously, $\mathscr{A}_{s}^{\prime} \subseteq \mathscr{A}_{w}^{\prime}$. If $\mathscr{A}$ is selfadjoint $\left(\mathscr{D}=\bigcap_{A \in \mathscr{A}} \mathscr{D}\left(A^{*}\right)\right)$, then $\mathscr{A}_{s}^{\prime}=\mathscr{A}_{w}^{\prime}$. We remark that there are also nonselfadjoint $\mathscr{O}^{*}$-algebras $\mathscr{A}$ such that $\mathscr{A}_{s}^{\prime}=\mathscr{A}_{w}^{\prime}$ (see $\left.[6, \S 5.5]\right)$.

\section{Generated EXTENSIONS OF *-REPRESENTATIONS}

Borchers and Yngvason [1] and Schmüdgen [7] developed methods for the construction of special extensions of $*$-representations of $*$-algebras and called them induced extensions. We present a slightly modified method. The proofs of the propositions in this section proceed similarly as in Chapter 8.5 in [7]. Therefore we omit them.

Let $\pi$ be a $*$-representation of a $*$-algebra $\mathfrak{A}$ on $\mathscr{D}(\pi)$ in the Hilbert space $\mathscr{H}(\pi)$. Further let $\mathscr{H}_{1} \supseteq \mathscr{H}(\pi)$ be a larger Hilbert space and $\mathscr{M}_{1}$ be a subset of $\mathbb{B}\left(\mathscr{H}_{1}\right)$ with $I \in \mathscr{M}_{1}$. We identify $\mathscr{H}(\pi)$ with the subspace $\mathscr{H}(\pi) \oplus\{0\}$ of $\mathscr{H}_{1}$ and define the set

$$
\mathscr{M}_{1} \mathscr{D}(\pi):=\text { l.h. }\left\{X \varphi: X \in \mathscr{M}_{1} \text { and } \varphi \in \mathscr{D}(\pi)\right\} .
$$


Let $\mathscr{H}_{0}$ be the closure of $\mathscr{M}_{1} \mathscr{D}(\pi)$ in $\mathscr{H}_{1}$. Let $P$ and $Q$ denote the projections from $\mathscr{H}_{1}$ onto $\mathscr{H}_{0}$ and from $\mathscr{H}_{1}$ onto $\mathscr{H}(\pi)$, respectively. We define a surjective transformation $\varepsilon: \mathbb{B}\left(\mathscr{H}_{1}\right) \rightarrow \mathbb{B}\left(\mathscr{H}_{0}\right)$ by

$$
\varepsilon(X):=P X \mid \mathscr{H}_{0} \text { for all } X \in \mathbb{B}\left(\mathscr{H}_{1}\right) .
$$

Proposition 1. Let $\pi$ be a *-representation of the *-algebra $\mathfrak{A}$ on $\mathscr{D}(\pi)$ in the Hilbert space $\mathscr{H}(\pi)$ and let $\mathscr{H}_{1} \supseteq \mathscr{H}(\pi)$ be a larger Hilbert space. Suppose that $\mathscr{M}_{1}$ is a subset of $\mathbb{B}\left(\mathscr{H}_{1}\right)$ with $I \in \mathscr{M}_{1}$. Further let $\mathscr{H}_{0}$ be the closure of $\mathscr{M}_{1} \mathscr{D}(\pi)$ in $\mathscr{H}_{1}$. Suppose $Q\left(Y^{*} X\right) \mid \mathscr{H}(\pi) \in \pi(\mathfrak{A})_{w}^{\prime}$ for all $X, Y \in \mathscr{M}_{1}$. Then,

$$
\begin{gathered}
\mathscr{D}\left(\pi_{0}\right):=\mathscr{M}_{1} \mathscr{D}(\pi) \\
\pi_{0}(a)\left(\Sigma_{k} X_{k} \varphi_{k}\right):=\Sigma_{k} X_{k} \pi(a) \varphi_{k} \text { for all } a \in \mathfrak{A},
\end{gathered}
$$

where $\Sigma_{k} X_{k} \varphi_{k}$ with $X_{k} \in \mathscr{M}_{1}$ and $\varphi_{k} \in \mathscr{D}(\pi)$ is any element of $\mathscr{D}\left(\pi_{0}\right)$, defines a *-representation $\pi_{0}$ of $\mathfrak{A}$ on $\mathscr{D}\left(\pi_{0}\right)$ in the Hilbert space $\mathscr{H}_{0}$ which extends $\pi$.

Definition 2. Suppose the assumptions of Proposition 1 are satisfied. We call the *-representation $\pi_{0}$ of $\mathfrak{A}$ in the Hilbert space $\mathscr{H}_{0}$ defined by (3) the extension of $\pi$, which is generated by $\mathscr{M}_{1} \subseteq \mathbb{B}\left(\mathscr{H}_{1}\right)$. We denote this generated extension by $\left(\pi_{0}, \mathscr{M}_{1}\right)$.

Proposition 3. Suppose the assumptions of Proposition 1 are fulfilled. Let $\left(\pi_{0}\right.$, $\mathscr{M}_{1}$ ) be the generated extension of $\pi$ defined as in (3). Further let $\mathscr{M}:=$ $\left\{\varepsilon(X): X \in \mathscr{M}_{1}\right\}$. If $X Y$ is in $\mathscr{M}_{1}$ when $X$ and $Y$ are in $\mathscr{M}_{1}$, then $\mathscr{M} \subseteq$ $\pi_{0}(\mathfrak{A})_{s}^{\prime}$.

Remark. Suppose the assumptions of Proposition 3 are fulfilled. For any element $X \in \mathscr{M}_{1} \quad Q X\left\lceil\mathscr{H}(\pi) \in \pi(\mathfrak{A})_{w}^{\prime}\right.$ and $\varepsilon(X) \in \pi_{0}(\mathfrak{A})_{s}^{\prime}$. Consequently, some operators from the weak commutant of the starting $*$-representation $\pi$ turn into operators from the strong commutant of the final generated extension $\pi_{0}$. Therefore the method of generated extensions seems to be suitable to construct extensions such that the "difference" between strong and weak commutant "decreases."

Next we describe the bounded operators in the Hilbert space $\dot{\mathscr{H}}_{0}$ via bounded operators in $\mathscr{H}_{1}$. We define the set

$$
\mathbb{B}_{\mathscr{K}_{0}}\left(\mathscr{H}_{1}\right):=\left\{X \in \mathbb{B}\left(\mathscr{H}_{1}\right): X \mid \mathscr{H}_{1} \ominus \mathscr{H}_{0}=0 \text { and } X \mathscr{H}_{0} \subseteq \mathscr{H}_{0}\right\} .
$$

With the projection $P$ from $\mathscr{H}_{1}$ onto $\mathscr{H}_{0}$ we get

$$
\mathbb{B}_{\mathscr{H}_{0}}\left(\mathscr{H}_{1}\right)=\left\{X \in \mathbb{B}\left(\mathscr{H}_{1}\right): X=P X P\right\} .
$$

The transformation $\varepsilon \mid \mathbb{B}_{\mathscr{H}_{0}}\left(\mathscr{H}_{1}\right)$ (see (2)) is a bijection from $\mathbb{B}_{\mathscr{P}_{0}}\left(\mathscr{H}_{1}\right)$ onto $\mathbb{B}\left(\mathscr{H}_{0}\right)$. Now it is possible to describe the weak commutant of generated extensions. 
Proposition 4. Suppose the assumptions of Proposition 1 are fulfilled. Let $\left(\pi_{0}\right.$, $\left.\mathscr{M}_{1}\right)$ be the generated extension of $\pi$ defined as in (3). Then,

$$
\begin{array}{r}
\pi_{0}(\mathfrak{A})_{w}^{\prime}=\left\{\varepsilon(Z): Z \in \mathbb{B}_{\mathscr{H}_{0}}\left(\mathscr{H}_{1}\right) \text { and } Q\left(Y^{*} Z X\right) \mid \mathscr{H}(\pi) \in \pi(\mathfrak{A})_{w}^{\prime}\right. \\
\text { for all } \left.X, Y \in \mathscr{M}_{1}\right\} .
\end{array}
$$

\section{GENERATED EXTENSIONS OF SPECIAL *-REPRESENTATIONS}

In this section we consider especially $*$-representations of the $*$-algebra $\mathfrak{A}:=$ $\mathscr{P}(x)$ of all complex polynomials in one hermitian variable $x$ endowed with the usual algebraic operations.

Let $A$ be a closed symmetric operator in a Hilbert space $\mathscr{H}$ with at least one deficiency index being finite. By Theorem 1.9 in [5], $\mathscr{D}^{\infty}(A):=\bigcap_{n \in \mathbb{N}} \mathscr{D}\left(A^{n}\right)$ is dense in $\mathscr{H}$ and $\mathscr{D}^{\infty}(A)$ is a core for each power $A^{n}, n \in \mathbb{N}$. Hence

$$
\mathscr{D}(\pi):=\mathscr{D}^{\infty}(A), \quad \pi(x):=A \uparrow \mathscr{D}(\pi)
$$

defines a $*$-representation $\pi$ of $\mathfrak{A}$ in the Hilbert space $\mathscr{H}$.

Remarks. 1. The *-representation $\pi$ defined by (5) is closed. Indeed, $\bigcap_{p \in \mathscr{P}(x)} \mathscr{D}(\overline{\pi(p(x))}) \subseteq \bigcap_{n \in \mathbb{N}} \mathscr{D}\left(\overline{\pi\left(x^{n}\right)}\right)=\bigcap_{n \in \mathbb{N}} \mathscr{D}\left(A^{n}\right)=\mathscr{D}(\pi)$.

2. The commutants of the $\mathscr{O}^{*}$-algebra $\pi(\mathscr{P}(x))$ are given by

$$
\pi(\mathscr{P}(x))_{s}^{\prime}=(A)_{s}^{\prime} \text { and } \pi(\mathscr{P}(x))_{w}^{\prime}=(A)_{w}^{\prime} .
$$

Now we study generated extensions of $*$-representations $\pi$ defined as in (5). Let $\mathscr{H}_{1}:=\mathscr{H} \oplus \mathscr{H}$, and let $T:=\left[\begin{array}{ll}T_{1} & 0 \\ T_{2} & I\end{array}\right]$ with $T_{1}, T_{2} \in \mathbb{B}(\mathscr{H})$ be an operator from $\mathbb{B}\left(\mathscr{H}_{1}\right)$ with the additional conditions

$$
T_{1} \in \pi(\mathfrak{A})_{w}^{\prime} \quad \text { and } \quad T_{1}^{*} T_{1}+T_{2}^{*} T_{2} \in \pi(\mathfrak{A})_{w}^{\prime} .
$$

Further let $\mathscr{M}_{1}:=\{T, I\} \subset \mathbb{B}\left(\mathscr{H}_{1}\right)$. By Proposition 1,

$$
\begin{aligned}
& \mathscr{D}\left(\pi_{T}\right):=\left\{\left(\begin{array}{c}
\varphi+T_{1} \psi \\
T_{2} \psi
\end{array}\right): \varphi, \psi \in \mathscr{D}(\pi)\right\} \\
& \pi_{T}(a)\left(\begin{array}{c}
\varphi+T_{1} \psi \\
T_{2} \psi
\end{array}\right):=\left(\begin{array}{c}
\pi(a) \varphi+T_{1} \pi(a) \psi \\
T_{2} \pi(a) \psi
\end{array}\right),
\end{aligned}
$$

for all $a \in \mathfrak{A}$ and $\varphi, \psi \in \mathscr{D}(\pi)$,

defines a generated extension $\left(\pi_{T},\{T, I\}\right)$ of $\pi$ in the Hilbert space $\mathscr{H}_{T}:=$ $\overline{\mathscr{D}(\pi)+T \mathscr{D}(\pi)} \subseteq \mathscr{H}_{1}$. One easily checks that

$$
\mathscr{H}_{T}=\mathscr{H} \oplus \overline{\mathscr{R}\left(T_{2}\right)} \text {. }
$$

Let $E$ be the projection in $\mathscr{H}$ onto $\overline{\mathscr{R}\left(T_{2}\right)}$. By (4),

$$
\begin{array}{r}
\mathbb{B}_{\mathscr{H}_{T}}\left(\mathscr{H}_{1}\right)=\left\{\left[\begin{array}{ll}
Z_{11} & Z_{12} \\
Z_{21} & Z_{22}
\end{array}\right]: Z_{i j} \in \mathbb{B}(\mathscr{H}) \text { and } Z_{12} E=Z_{12},\right. \\
\left.E Z_{21}=Z_{21}, E Z_{22} E=Z_{22}\right\} .
\end{array}
$$


The identification with operators in $\mathbb{B}\left(\mathscr{\ell}_{T}\right)$ via the transformation $\varepsilon$ is given by

$$
\mathbb{B}_{\mathscr{H}_{T}}\left(\mathscr{H}_{1}\right) \ni\left[\begin{array}{ll}
Z_{11} & Z_{12} \\
Z_{21} & Z_{22}
\end{array}\right] \rightarrow\left[\begin{array}{ll}
Z_{11} & Z_{12} \\
Z_{21} & Z_{22}
\end{array}\right]\left\lceil\mathscr{H}_{T} \in \mathbb{B}\left(\mathscr{H}_{T}\right)\right. \text {. }
$$

We determine the commutants of a generated extension in a special situation.

Example 5. Let $\mathbb{T}:=\{z \in \mathbb{C}:|z|=1\}$ be the unit circle and $\mathscr{H}:=H^{2}(\mathbb{T})$ the Hardy space as a subspace of $L^{2}(\mathbb{T})$. We consider the closed symmetric operator $A:=i(I+S)(I-S)^{-1}$ on $\mathscr{D}(A)=(I-S) \mathscr{H}$, where $S$ is the unilateral shift. Let $\pi$ be the related $*$-representation of $\mathfrak{A}:=\mathscr{P}(x)$ defined as in (5). By Example 1.10 in [6] and the relation (6), we have $\pi(\mathfrak{A})_{s}^{\prime}=\left\{T_{\varphi}: \varphi \in H^{\infty}(\mathbb{T})\right\}$ and $\pi(\mathfrak{A})_{w}^{\prime}=\left\{T_{\varphi}: \varphi \in L^{\infty}(\mathbb{T})\right\}$. For $\varphi \in L^{\infty}(\mathbb{T}) T_{\varphi}$ denotes the Toeplitz operator defined by $T_{\varphi} f=P_{+}(\varphi f)$ for all $f \in H^{2}(\mathbb{T}) . P_{+}$is the projection from $L^{2}(\mathbb{T})$ onto $H^{2}(\mathbb{T})$. Further $H^{\infty}(\mathbb{T}):=L^{\infty}(\mathbb{T}) \cap H^{2}(\mathbb{T})$.

Let $\mathscr{H}_{1}:=\mathscr{H} \oplus \mathscr{H}$ and $T:=\left[\begin{array}{cc}s^{*} & 0 \\ s s^{*}+c S-I & I\end{array}\right] \in \mathbb{B}\left(\mathscr{H}_{1}\right)$ with $c \in \mathbb{R}, c \neq 0$. The operators $T_{1}=S^{*}$ and $T_{2}=S S^{*}+c S-I$ satisfy the conditions in (7). We have even $T_{1}^{*} T_{1}+T_{2}^{*} T_{2}=\left(c^{2}+1\right) I \in \pi(\mathfrak{A})_{s}^{\prime}$.

Let $\pi_{T}$ be the $*$-representation of $\mathscr{P}(x)$ given by $(8) . \mathscr{R}\left(T_{2}\right)$ is closed and $\mathscr{R}\left(T_{2}\right)^{\perp}$ is a one-dimensional subspace of $\mathscr{H}$. We find $\left(z^{1}+c z^{0}\right) \perp \mathscr{R}\left(T_{2}\right)$, and hence $E=I-\frac{1}{c^{2}+1}(S+c I)\left(I-S S^{*}\right)\left(S^{*}+c I\right)$, and $\mathscr{H}_{T}=\left\{\left(\begin{array}{l}\xi \\ \zeta\end{array}\right) \in \mathscr{H}_{1}: \xi \in \mathscr{H}\right.$ and $\left.\zeta \perp\left(z^{1}+c z^{0}\right)\right\}$. After some calculations we get for the commutants of the generated extension $\left(\pi_{T},\{T, I\}\right)$ :

$$
\begin{array}{r}
\pi_{T}(\mathfrak{A})_{w}^{\prime}=\left\{\begin{array}{cc}
T_{\alpha} & \frac{1}{c}\left(T_{\beta}-T_{\alpha} S^{*}\right) S^{*} E \\
\frac{1}{c} E S\left(T_{\gamma}-S T_{\alpha}\right) & \frac{1}{c^{2}} E S\left(S T_{\alpha} S^{*}-S T_{\beta}-T_{\gamma} S^{*}+T_{\delta}\right) S^{*} E
\end{array}\right] \mid \mathscr{H}_{T}: \\
\left.\alpha, \beta, \gamma, \delta \in L^{\infty}(\mathbb{T})\right\}
\end{array}
$$

and

$$
\begin{aligned}
\pi_{T}(\mathfrak{A})_{s}^{\prime}=\left\{\begin{array}{cc}
T_{\alpha} & \frac{1}{c}\left(T_{\beta}-T_{\alpha} S^{*}\right) S^{*} E \\
\frac{1}{c} E S\left(T_{\gamma}-S T_{\alpha}\right) & \frac{1}{c^{2}} E S\left(S T_{\alpha} S^{*}-S T_{\beta}-T_{\gamma} S^{*}+T_{\delta}\right) S^{*} E
\end{array}\right] \mid \mathscr{H}_{T}: \\
\gamma, \delta \in H^{\infty}(\mathbb{T}) \text { and } \alpha, \beta \in L^{\infty}(\mathbb{T}), \\
\left.\left(c^{2}+1\right) \alpha-\frac{1}{z} \gamma \in H^{\infty}(\mathbb{T}) \text { and }\left(c^{2}+1\right) \beta-\frac{1}{z} \delta \in H^{\infty}(\mathbb{T})\right\} .
\end{aligned}
$$

The "difference" between $\pi_{T}(\mathfrak{A})_{s}^{\prime}$ and $\pi_{T}(\mathfrak{A})_{w}^{\prime}$ is not maximal in the sense that $\left[\begin{array}{ll}Z_{11} & Z_{12} \\ Z_{21} & Z_{22}\end{array}\right] \mid \mathscr{H}_{T} \in \pi_{T}(\mathfrak{A})_{s}^{\prime}$ does not imply $\alpha, \beta, \gamma, \delta \in H^{\infty}(\mathbb{T})$.

\section{ANOTHER METHOD TO EXTEND *-REPRESENTATIONS}

In this section let $\mathfrak{A}$ be a $*$-algebra with unit 1 and let $\mathfrak{B}$ be a subset of its hermitian part $\mathfrak{A}_{h}$ such that $\mathfrak{A}$ is generated by $\mathfrak{B} \cup\{1\}$ as an algebra. That is, we suppose that $\mathfrak{A}$ is of the form

$$
\mathfrak{A}=\text { l.h. }\left\{b_{1} \cdots b_{k}: k \in \mathbb{N}_{0}, b_{1}, \ldots, b_{k} \in \mathfrak{B}\right\} .
$$


Let $\pi$ be a $*$-representation of $\mathfrak{A}$ on $\mathscr{D}(\pi)$ in a Hilbert space $\mathscr{H}(\pi)$. We present an extension method that is closely related with the extension of the symmetric operators $\pi(b), b \in \mathfrak{B}$. We need the following lemma proved in Chapter 8.1 in [7].

Lemma 6. Let $\pi$ be a *-representation of $\mathfrak{A}$ being of the form (11). Then,

$$
\mathscr{D}\left(\pi^{*}\right)=\bigcap_{k \in \mathbb{N} b_{1}, \ldots, b_{k} \in \mathfrak{B}} \mathscr{D}\left(\pi\left(b_{k}\right)^{*} \cdots \pi\left(b_{1}\right)^{*}\right) .
$$

The extension method is based on the following Proposition. Its proof is similar to the proof of Proposition 8.1.17 in [7].

Proposition 7. Suppose $\mathfrak{A}$ is a *-algebra with 1 and $\mathfrak{B} \subseteq \mathfrak{A}_{h}$ is such that $\mathfrak{A}$ is generated by $\mathfrak{B} \cup\{1\}$ as an algebra. Let $\pi$ be $a$ *-representation of $\mathfrak{A}$ on $\mathscr{D}(\pi)$ in the Hilbert space $\mathscr{H}(\pi)$. Suppose that there exist selfadjoint extensions $S_{b} \supseteq \pi(b)$ in the Hilbert space $\mathscr{H}(\pi)$ for all $b \in \mathfrak{B}$. Further let

$$
\left.\mathscr{D}_{0}:=\bigcap_{k \in \mathbb{N} b_{1}, \ldots, b_{k} \in \mathfrak{B}} \bigcap_{b_{1}} \cdots S_{b_{k}}\right) \text {. }
$$

(i) Then $\mathscr{D}_{0} \subseteq \mathscr{D}\left(\pi^{*}\right)$ and $\pi_{0}:=\pi^{*} \uparrow \mathscr{D}_{0}$ is a closed *-representation of $\mathfrak{A}$ with $\pi_{0} \supseteq \pi$.

(ii) If $\mathscr{D}_{0}$ is a core for all $S_{b}, b \in \mathfrak{B}$, then $\pi_{0}$ is a selfadjoint *-representation of $\mathfrak{A}$.

Proof. (i) For all $b \in \mathfrak{B}$ we have $S_{b} \subseteq \pi(b)^{*}$. Let $b_{1}, \ldots, b_{k} \in \mathfrak{B}$. Then $S_{b_{1}} \cdots S_{b_{k}} \subseteq \pi\left(b_{1}\right)^{*} \cdots \pi\left(b_{k}\right)^{*} \subseteq\left(\pi\left(b_{k}\right) \cdots \pi\left(b_{1}\right)\right)^{*}=\pi\left(b_{k} \cdots b_{1}\right)^{*}$. From the general definition of $\mathscr{D}\left(\pi^{*}\right)$ we conclude $\mathscr{D}_{0} \subseteq \mathscr{D}\left(\pi^{*}\right)$. Since $S_{b} \mathscr{D}_{0} \subseteq \mathscr{D}_{0}$ for all $b \in \mathfrak{B}$, we have $\pi(b)^{*} \mathscr{D}_{0} \subseteq \mathscr{D}_{0}$ for all $b \in \mathfrak{B}$. Since $\mathfrak{B} \cup\{1\}$ generates $\mathfrak{A}$ as an algebra and $\pi^{*}$ is a homomorphism, the latter implies $\pi(a)^{*} \mathscr{D}_{0} \subseteq \mathscr{D}_{0}$ for all $a \in \mathfrak{A}$. Being the restriction of the representation $\pi^{*}$ to the invariant domain $\mathscr{D}_{0}, \pi_{0}$ is a representation of $\mathfrak{A}$.

Moreover, for all $b \in \mathfrak{B}$ and $\varphi, \psi \in \mathscr{D}_{0}\left\langle\pi_{0}(b) \varphi, \psi\right\rangle=\left\langle\pi(b)^{*} \varphi, \psi\right\rangle=$ $\left\langle S_{b} \varphi, \psi\right\rangle=\left\langle\varphi, S_{b} \psi\right\rangle=\left\langle\varphi, \pi(b)^{*} \psi\right\rangle=\left\langle\varphi, \pi_{0}(b) \psi\right\rangle$. Hence $\pi_{0}$ is even a *representation of $\mathfrak{A}$. From $\mathscr{D}(\pi) \subseteq \mathscr{D}_{0}$ we conclude $\pi \subseteq \pi_{0}$.

We can show the completeness of $\mathscr{D}_{0}$ in the graph topology of $\pi_{0}(\mathfrak{A})$ in the same way as in Proposition 8.1.17 in [7] by induction. Hence $\pi_{0}$ is a closed *-representation.

(ii): Let $\mathscr{D}_{0}$ be a core for $S_{b}$ for all $b \in \mathfrak{B}$. From the proof of (i) we know that $\pi_{0}(b)=S_{b} \uparrow \mathscr{D}_{0}$ for all $b \in \mathfrak{B}$. Hence $\pi_{0}(b)^{*}=\left(S_{b} \uparrow \mathscr{D}_{0}\right)^{*}=S_{b}$ for all $b \in \mathfrak{B}$. Using (12) in Lemma 6, we get

$$
\begin{aligned}
\mathscr{D}\left(\pi_{0}^{*}\right) & =\bigcap_{k \in \mathbb{N} b_{1}, \ldots, b_{k} \in \mathfrak{B}} \bigcap_{\mathcal{D}\left(\pi_{0}\left(b_{k}\right)^{*} \cdots \pi_{0}\left(b_{1}\right)^{*}\right)} \mathscr{D}\left(S_{b_{k}} \cdots S_{b_{1}}\right)=\mathscr{D}_{0}=\mathscr{D}\left(\pi_{0}\right) .
\end{aligned}
$$

Hence $\pi_{0}$ is selfadjoint and the proof is complete. 
The extension method works as follows. Let $\pi$ be a $*$-representation of $\mathfrak{A}$ with $\operatorname{dim}((\pi(b)+i I) \mathscr{D}(\pi))^{\perp}=\operatorname{dim}((\pi(b)-i I) \mathscr{D}(\pi))^{\perp}$ for all $b \in \mathfrak{B}$. Then there exist selfadjoint extensions $S_{b}$ of $\pi(b)$ inside the Hilbert space $\mathscr{H}(\pi)$ for all $b \in \mathfrak{B}$. We define $\mathscr{D}_{0}$ as in (13) and get the closed *-representation $\pi_{0}=\pi^{*} \mid \mathscr{D}_{0}$ of $\mathfrak{A}$, which is an extension of $\pi$. If we can choose the selfadjoint extensions $S_{b}$ for all $b \in \mathfrak{B}$ such that $\mathscr{D}_{0}$ is a core for all $S_{b}, b \in \mathfrak{B}$, then $\pi_{0}$ is a selfadjoint extension of $\pi$. Then especially the commutants $\pi_{0}(\mathfrak{A})_{s}^{\prime}$ and $\pi_{0}(A)_{w}^{\prime}$ of the $\mathscr{O}^{*}$-algebra $\pi_{0}(\mathfrak{A})$ coincide.

In general we have for $b \in \mathfrak{B}$

$$
\operatorname{dim}((\pi(b)+i I) \mathscr{D}(\pi))^{\perp} \neq \operatorname{dim}((\pi(b)-i I) \mathscr{D}(\pi))^{\perp} .
$$

If the deficiency indices of $\overline{\pi(b)}$ are different for at least one $b \in \mathfrak{B}$, then we consider instead of the *-representation $\pi$ of $\mathfrak{A}$ on $\mathscr{D}(\pi)$ in the Hilbert space $\mathscr{H}(\pi)$ the $*$-representation $\tilde{\pi}:=\pi \oplus(-\pi)$ of $\mathfrak{A}$ on $\mathscr{D}(\tilde{\pi}):=\mathscr{D}(\pi) \oplus \mathscr{D}(\pi)$ in the Hilbert space $\mathscr{H}(\tilde{\pi}):=\mathscr{H}(\pi) \oplus \mathscr{H}(\pi)$. Then, $\operatorname{dim}((\tilde{\pi}(b)+i I) \mathscr{D}(\tilde{\pi}))^{\perp}=$ $\operatorname{dim}((\tilde{\pi}(b)-i I) \mathscr{D}(\tilde{\pi}))^{\perp}$ for all $b \in \mathfrak{B}$. Now we go on as above.

This method is useful for $*$-representations of the $*$-algebras $\mathscr{P}\left(x_{1}, \ldots, x_{n}\right)$ of all complex polynomials in $n$ commuting hermitian variables, for $*$-representations of the Weyl-algebras $\mathscr{A}\left(p_{1}, q_{1}, \ldots, p_{n}, q_{n}\right)$ and more generally for *-representations of the enveloping algebra $\mathfrak{E}(g)$ of a finite dimensional Lie algebra $g$.

Example 8. Let $\mathfrak{A}:=\mathscr{P}(x)$. Then $\mathfrak{B}:=\{x\}$ is a subset of $\mathfrak{A}_{h}$ such that $\mathfrak{B} \cup\{1\}$ generates $\mathfrak{A}$ as an algebra.

Let $A$ be a closed symmetric operator in a Hilbert space $\mathscr{H}$ with at least one deficiency index being finite. We consider the *-representation $\pi$ of $\mathfrak{A}$ defined by (5). Without loss of generality we assume that the deficiency indices of $A$ coincide. Otherwise we consider the $*$-representation $\pi \oplus(-\pi)$ instead of $\pi$.

Let $B$ be any selfadjoint extension of $A$ in $\mathscr{H}$. With $S_{x}:=B$ we get for $\mathscr{D}_{0}$ defined by (13) $\mathscr{D}_{0}=\bigcap_{n \in \mathbb{N}} \mathscr{D}\left(B^{n}\right)=\mathscr{D}^{\infty}(B)$. Again by Theorem 1.9 in [5], $\mathscr{D}_{0}$ is a core for $B$. By Proposition 7, the $*$-representation $\pi_{0}$ defined by

$$
\mathscr{D}\left(\pi_{0}\right):=D^{\infty}(B), \quad \pi_{0}(x):=B \mid \mathscr{D}\left(\pi_{0}\right)
$$

is a selfadjoint extension of $\pi$.

\section{AN EXAMPLE}

In this section we show that the method of generated extensions can be used to extend a given $*$-representation such that the commutants of the related $\mathscr{O}^{*}$ algebra coincide. We start as in Example 5 with the Hardy space $H^{2}(\mathbb{T})=$ : $\mathscr{H}$ and the closed symmetric operator $A:=i(I+S)(I-S)^{-1}$ with $\mathscr{D}(A)=$ $(I-S) \mathscr{H}$. Let $\mathfrak{A}:=\mathscr{P}(x)$ and let $\pi$ be the related $*$-representation of $\mathfrak{A}$ defined by

$$
\mathscr{D}(\pi):=\mathscr{D}^{\infty}(A), \quad \pi(x):=A \uparrow \mathscr{D}(\pi) .
$$


Then we have $\pi(\mathfrak{A})_{s}^{\prime}=\left\{T_{\varphi}: \varphi \in H^{\infty}(\mathbb{T})\right\}$ and $\pi(\mathfrak{A})_{w}^{\prime}=\left\{T_{\varphi}: \varphi \in L^{\infty}(\mathbb{T})\right\}$. Hence $\pi(\mathfrak{A})_{s}^{\prime} \neq \pi(\mathfrak{A})_{w}^{\prime}$.

First, we construct a selfadjoint extension $B$ of $A$ in a larger Hilbert space. The operator $\widetilde{A}:=A \oplus(-A)$ in the Hilbert space $\mathscr{H} \oplus \mathscr{H}$ has the deficiency indices $(1,1)$. We have $\mathscr{R}(\tilde{A}+i I)=\mathscr{H} \oplus S \mathscr{H}$ and $\mathscr{R}(\tilde{A}-i I)=S \mathscr{H} \oplus \mathscr{H}$. The Cayley transform $\widetilde{U}$ as a partial isometry with the initial space $\mathscr{R}(\widetilde{A}+i I)$ is defined by $\widetilde{U}(\varphi, S \psi)=(S \varphi, \psi)$ for all $\varphi, \psi \in \mathscr{H}$. One easily checks that the operator $U$ defined by $U(\varphi, \psi)=\left(S \varphi+\left(I-S S^{*}\right) \psi, S^{*} \psi\right)$ for all $\varphi, \psi \in \mathscr{H}$ is a unitary extension of $\widetilde{U}$. Let $\mathrm{B}$ be the selfadjoint extension of $\tilde{A}$ in $\mathscr{H} \oplus \mathscr{H}$ whose Cayley transform is $U$. Hence $\mathscr{D}(B)=(I-U)(\mathscr{H} \oplus \mathscr{H})$ and $B(I-U)(\varphi, \psi)=i(I+U)(\varphi, \psi)$ for all $\varphi, \psi \in \mathscr{H}$.

Now let $\pi_{1}$ be the *-representation of $\mathfrak{A}$ in the Hilbert space $\mathscr{H} \oplus \mathscr{H}$ given by

$$
\mathscr{D}\left(\pi_{1}\right):=\mathscr{D}^{\infty}(B), \quad \pi_{1}(x):=B \nmid \mathscr{D}\left(\pi_{1}\right) .
$$

According to Example 8, $\pi_{1}$ is a selfadjoint extension of $\pi$. Our aim is to construct generated extensions of $\pi$ that are closely related to the selfadjoint extension $\pi_{1}$. To do this we are looking for suitable operators $T=\left[\begin{array}{c}T_{1} \\ T_{2}\end{array}\right] \in$ $\mathbb{B}(\mathscr{H} \oplus \mathscr{H})$.

Let $\Phi \in H^{\infty}(\mathbb{T})$ be a cyclic vector for $S^{*}$. This means $H^{2}(\mathbb{T})=$ c.l.h. $\left\{\left(S^{*}\right)^{n} \Phi: n \in \mathbb{N}_{0}\right\}$. For example $\Phi(z)=e^{z}$ is such a function (see [2, Lect. II.1]). Define $\widetilde{\Phi}(z):=\Phi(\bar{z})$. Let $T_{1}:=T_{z \Phi+z \tilde{\Phi}}$ be the Toeplitz operator in $\mathscr{H}$ with the symbol $z \Phi+\bar{z} \widetilde{\Phi} \in L^{\infty}(\mathbb{T})$ and $T_{2}:=P_{+} J M_{\widetilde{\Phi}}$, where $M_{\widetilde{\Phi}}$ denotes the operator of multiplication by $\widetilde{\Phi}$ and $J$ is the flip operator on $L^{2}(\mathbb{T})$ given by $J f(z)=f(\bar{z})$. The operator $T_{2} \in \mathbb{B}(\mathscr{H})$ is a Hankel operator (see [3]).

Lemma 9. The operators $T_{1}$ and $T_{2}$ have the following properties:

(i) $T_{1} \in \pi(\mathfrak{A})_{w}^{\prime}$

(ii) $T_{1}^{*} T_{1}+T_{2}^{*} T_{2} \in \pi(\mathfrak{A})_{w}^{\prime}$

(iii) $T_{2} S=S^{*} T_{2}$

(iv) $T_{1} S=S T_{1}+\left(I-S S^{*}\right) T_{2}$.

The proof is straightforward and therefore omitted. Now let $T:=\left[\begin{array}{c}T_{1} \\ T_{2} \\ I\end{array}\right]$. Because of Lemma 9, (i) and (ii), the *-representation $\pi_{T}$ defined by (8) is the generated extension $\left(\pi_{T},\{T, I\}\right)$ of $\pi$. Since $\Phi$ is cyclic for $S^{*}$ and $T_{2} z^{n}=$ $\left(S^{*}\right)^{n} \Phi$ for all $n \in \mathbb{N}_{0}$, we find $\overline{\mathscr{R}\left(T_{2}\right)}=\mathscr{H}$. Hence by $(9), \mathscr{H}_{T}=\mathscr{H} \oplus \mathscr{H}$. By Lemma 9, (iii) and (iv), we find $T S \varphi=U T \varphi$ for all $\varphi \in \mathscr{H}$. Now we can prove the following result.

Proposition 10. Let $\left(\pi_{T},\{T, I\}\right)$ be a generated extension of $\pi$ in the Hilbert space $\mathscr{H} \oplus \mathscr{H}$ constructed above. Then,

(i) $\pi_{T} \subseteq \pi_{1}$;

(ii) the weak commutant $\pi_{T}(\mathfrak{A})_{w}^{\prime}$ is an algebra; and

(iii) $\pi_{T}^{*}=\pi_{1}$. That is, $\pi_{T}^{*}$ is selfadjoint. 
Proof. (i) Suppose $\xi, \zeta$ are any vectors from $\mathscr{D}(\pi)=\mathscr{D}^{\infty}(A)$. Then $\xi+T \zeta$ is any element of $\mathscr{D}\left(\pi_{T}\right)$. Let $n \in \mathbb{N}$. Since $\mathscr{D}(\pi) \subseteq \mathscr{D}\left(A^{n}\right)$ and $\mathscr{D}\left(A^{n}\right)=$ $(I-S)^{n} \mathscr{H}$ (see [5, Lemma 1.5]), there exist vectors $\varphi, \psi \in \mathscr{H}$ such that $\xi=(I-S)^{n} \varphi$ and $\zeta=(I-S)^{n} \psi$. Then we get $\xi+T \zeta=(I-S)^{n} \varphi+$ $T(I-S)^{n} \psi=(I-U)^{n}(\varphi+T \psi) \in \mathscr{D}\left(B^{n}\right)$, and $\pi_{T}\left(x^{n}\right)(\xi+T \zeta)=A^{n} \xi+$ $T A^{n} \zeta=(i)^{n}(I+S)^{n} \varphi+(i)^{n} T(I+S)^{n} \psi=(i)^{n}(I+U)^{n}(\varphi+T \psi)=B^{n}(\xi+T \zeta)$. Hence for all $n \in \mathbb{N}, \mathscr{D}\left(\pi_{T}\right) \subseteq \mathscr{D}\left(B^{n}\right)$ and consequently, $\mathscr{D}\left(\pi_{T}\right) \subseteq \mathscr{D}\left(\pi_{1}\right)$. Moreover, $\pi_{1}\left(x^{n}\right) \uparrow \mathscr{D}\left(\pi_{T}\right)=B^{n} \uparrow \mathscr{D}\left(\pi_{T}\right)=\pi_{T}\left(x^{n}\right)$. This Implies $\pi_{T} \subseteq \pi_{1}$.

(ii) We show that $\mathscr{D}\left(\pi_{T}\right)=\mathscr{D}(\pi)+T \mathscr{D}(\pi)$ is a core for $B$. First, $\mathscr{D}(\pi)=$ $(I-S) \mathscr{D}(\pi)$. Hence $\mathscr{D}\left(\pi_{T}\right)=(I-U) \mathscr{D}\left(\pi_{T}\right)$. Then $(B+i I) \mathscr{D}\left(\pi_{T}\right)=\mathscr{D}\left(\pi_{T}\right)$ and $(B-i I) \mathscr{D}\left(\pi_{T}\right)=U \mathscr{D}\left(\pi_{T}\right)$. Since $\mathscr{D}\left(\pi_{T}\right)$ and $U \mathscr{D}\left(\pi_{T}\right)$ are dense in $\mathscr{H} \oplus \mathscr{H}, B \uparrow \mathscr{D}\left(\pi_{T}\right)$ is essentially selfadjoint. Thus $\mathscr{D}\left(\pi_{T}\right)$ is a core for $B$. This implies that the weak commutant of the $\mathscr{O}^{*}$-algebra $\pi_{T}(\mathscr{P}(x))$ is an algebra (see for instance [4, Lemma 3.2]).

(iii) From $\pi_{T}(x)=B \mid \mathscr{D}\left(\pi_{T}\right)$ and the fact that $\mathscr{D}\left(\pi_{T}\right)$ is a core for $B$, we get $\pi_{T}(x)^{*}=B$. By Lemma 6, $\mathscr{D}\left(\pi_{T}^{*}\right)=\bigcap_{n \in \mathbb{N}} \mathscr{D}\left(\left(\pi_{T}(x)^{*}\right)^{n}\right)=\bigcap_{n \in \mathbb{N}} \mathscr{D}\left(B^{n}\right)=$ $\mathscr{D}\left(\pi_{1}\right)$. Hence $\pi_{T}^{*}=\pi_{1}$ and the proof is complete.

We close this section with the following essential remark.

Remark. There exists a generated extension $\pi_{0}$ of $\pi_{T}$ in the same Hilbert space such that $\pi_{0}(\mathfrak{A})_{s}^{\prime}=\pi_{0}(\mathfrak{A})_{w}^{\prime}$. Indeed, let $\mathscr{H}_{1}:=\mathscr{H} \oplus \mathscr{H}$ and $\mathscr{M}_{1}:=\pi_{T}(\mathfrak{A})_{w}^{\prime}$, which is an algebra by Proposition 10, (ii). We define the generated extension $\left(\pi_{0}, \mathscr{M}_{1}\right)$ as in (3). By Proposition 3, then $\pi_{T}(\mathfrak{A})_{w}^{\prime} \subseteq \pi_{0}(\mathfrak{A})_{s}^{\prime}$. On the other hand by $\pi_{T} \subseteq \pi_{0}$, we have $\pi_{0}(\mathfrak{A})_{s}^{\prime} \subseteq \pi_{0}(\mathfrak{A})_{w}^{\prime} \subseteq \pi_{T}(\mathfrak{A})_{w}^{\prime}$. Thus $\pi_{0}(\mathfrak{A})_{s}^{\prime}=\pi_{0}(\mathfrak{A})_{w}^{\prime}$.

\section{ACKNOWLEDGMENT}

The author would like to thank Professor K. Schmüdgen for numerous and valuable discussions.

\section{REFERENCES}

1. H. J. Borchers and J. Yngvason, On the algebra of field operators. The weak commutant and integral decomposition of states, Comm. Math. Phys. 42 (1975), 231-252.

2. N. K. Nikol' skii, Treatise on the shift operator, Springer-Verlag, Berlin, New York, 1986.

3. S. C. Power, Hankel operators on Hilbert space, Bull. London Math. Soc. 12 (1980), 422-442.

4. R. T. Powers, Selfadjoint algebras of uniounded operators, Comm. Math. Phys. 21 (1971), 85-124.

5. K. Schmüdgen, On domains of powers of closed symmetric operators, J. Operator Theory 9 (1983), 53-75.

6. Unbounded commutants and intertwining spaces of unbounded symmetric operators and *-representations, J. Funct. Anal. 71 (1987), 47-68.

7. __ Unbounded operator algebras and representations, Akademie-Verlag, Berlin, 1989.

Sektion Mathematik, Karl-Marx-Universität Leipzig, 7010 Leipzig, German DemoCRATIC REPUBLIC 